\title{
Conditional survival estimate of acute-on-chronic hepatitis B liver failure: A dynamic prediction based on a multicenter cohort
}

\author{
Ming-Hua Zheng ${ }^{1,2}$, Sheng-Jie Wu ${ }^{3}$, Ke-Qing Shi, ${ }^{1,2}$ Hua-Dong Yan ${ }^{4}$, Hai $\mathbf{L i}^{5}$, \\ Gui-Qi Zhu ${ }^{1,6}$, Yao-Yao Xie ${ }^{7}$, Fa-Ling Wu ${ }^{1,2}$, Yong-Ping Chen ${ }^{1,2}$ \\ ${ }^{1}$ Department of Infection and Liver Diseases, Liver Research Center, The First Affiliated Hospital of Wenzhou Medical \\ University, Wenzhou 325000, China \\ ${ }^{2}$ Institute of Hepatology, Wenzhou Medical University, Wenzhou 325000, China \\ ${ }^{3}$ Department of Cardiovascular Medicine, The Heart Center, The First Affiliated Hospital of Wenzhou Medical University, \\ Wenzhou 325000, China \\ ${ }^{4}$ Department of Infectious Diseases, Ningbo 315010, China \\ ${ }^{5}$ Department of Intensive Care Unit, Tianjin Infectious Disease Hospital, Tianjin 300000, China \\ ${ }^{6}$ School of the First Clinical Medical Sciences, Wenzhou Medical University, Wenzhou 325000, China \\ ${ }^{7}$ Department of Clinical Laboratory, The First Affiliated Hospital of Wenzhou Medical University, Wenzhou 325000, China \\ Correspondence to: \\ Yong-Ping Chen, e-mail: 13505777281@163.com \\ Keywords: acute-on-chronic hepatitis B liver failure, conditional survival, relative survival, prognosis, risk factor \\ Received: May 19, $2015 \quad$ Accepted: July 02, 2015 Published: July 15, 2015
}

\section{ABSTRACT}

Objectives: Counseling patients with acute-on-chronic hepatitis B liver failure (ACHBLF) on their individual risk of short-term mortality is challenging. This study aimed to develop a conditional survival estimate (CSE) for predicting individualized mortality risk in ACHBLF patients.

Methods: We performed a large prospective cohort study of 278 ACHBLF patients from December 2010 to December 2013 at three participating medical centers. The Kaplan-Meier method was used to calculate the cumulative overall survival (OS). Cox proportional hazard regression models were used to analyze the risk factors associated with OS. 4-week CSE at " $X$ " week after diagnostic established were calculated as $\mathrm{CS}_{4}=0 \mathrm{SS}_{(\mathrm{x}+4)} / \mathrm{OS}_{(\mathrm{x})}{ }^{*}$

Results: The actual OS at 2, 4, 6, 8, 12 weeks were $80.5 \%, 71.8 \%, 69.3 \%$, $66.0 \%$ and $63.7 \%$, respectively. Using CSE, the probability of surviving an additional 4 weeks, given that the patient had survived for $1,3,5,7,9$ weeks was $74 \%, 86 \%$, $92 \%, 93 \%, 97 \%$, respectively. Patients with worse prognostic feathers, including MELD > 25, Child grade C, age > 45, HE, INR > 2.5, demonstrated the greatest increase in CSE over time, when compared with the "favorable" one ( $\Delta 36 \%$ vs. $\Delta 10 \%$; $\Delta 28 \%$ vs. $\Delta 16 \% ; \Delta 29 \%$ vs. $\Delta 15 \% ; \Delta 60 \%$ vs. $\Delta 12 \% ; \Delta 33 \%$ vs. $\Delta 12 \% ;$ all $P<0.001 ;$ respectively).

Conclusions: This easy-to-use CSE can accurately predict the changing probability of survival over time. It may facilitate risk communication between patients and physicians.

\section{INTRODUCTION}

Acute-on-chronic liver failure (ACLF) is a critical clinical entity that occurs in patients with acute deterioration of diagnosed or undiagnosed chronic liver disease. It is accompanied with a high mortality rate ranging from
$32 \%$ to $68 \%$, which occurs mainly in the first 3 months once diagnosed [1-2]. In areas of high hepatitis B virus (HBV) prevalence, such as the developing countries in Asia, acuteon-chronic hepatitis B liver failure (ACHBLF) accounts for $>70 \%$ of ACLF and almost 120000 patients die per year [3-5]. Due to the unavailability of accurate, reliable and 
accessible screening tools for predicting which patients are at a borderline risk of worsening disease with an increased chance of fatal outcome, makes appropriate risk stratification and physician-patient communication challenging. Identifying patients at risk of disease progression to to death may help in early management decisions and in justifying health resource allocation [6].

Recently, considerable effort has been extended to earlier and/or more accurate methods to predict the prognosis of this specific clinical condition [7-11]. Most of the models and/or scoring systems are based on the variable of a single-point measurement, mainly at the point of diagnosis or during patient hospitalization. However, these static variables, invariably taken at a single-point in time may be characterized by poor sensitivity and specificity, especially during the early stages of the disease. A dynamic prediction of disease progression and outcome is urgently required $[4,12]$.

Survival estimates for ACHBLF are commonly reported as survival time following diagnosis. However, such survival estimates, based on conventional survival curves may not provide a real-time prediction for survival. This is largely due to the fact that the risk of death often is highest during the initial few weeks of follow-up after the date of diagnosis is established. Due to this reason, conditional survival estimate (CSE), which accounts for existing survival time has been proposed as a more relevant way to precisely predict the prognosis [12]. In recent years, CSE has been widely introduced in clinical oncology for clinical validation, including predicting the overall survival (OS) and disease-free survival of gastric cancer after surgical resection [13-15], metastatic renal-cell carcinoma [16-17], lung cancer [18-19], diffuse large B-cell lymphoma [20], pancreatic ductal adenocarcinoma [21] and breast cancer [22]. This type of exploration is in its early stage as the only study reported to date is in the field of cardiac failure [23]. As shown with above studies, CSEs are easy-to-use ladder diagrams for predicting the risk of an individual developing an outcome over a specified time period. Furthermore, CSE may provide a more "dynamic" or "real-time" estimate of the risk of death over time and is significantly different from traditional survival estimates [24]. In turn, CSE can be more helpful in tailoring patient-specific treatment, surveillance, and education based on individual survival characteristics.

The specific aim of the current study was to develop a simple and clinically useful CSE for assessing short-term mortality risk in a multicenter cohort of ACHBLF patients. Moreover, we also assessed the effect of various independent risk factors on OS and CSE among ACHBLF patients.

\section{RESULTS}

\section{Baseline characteristics}

A total of 278 cases were enrolled in this study. During the follow-up of $36.2 \pm 12.3$ months, 109 patients $(39.2 \%)$ died. The mean age was $45.8 \pm 13.2$ years, and the patients were predominantly men (77.3\%). The most common complication of ACHBLF was ascites (156 patients; 56.1\%) followed by LC (127 patients; 45.6\%), SBP (101 patients; 36.6\%), HE (57 patients; 20.5\%) and HRS (15 patients; 5.4\%) (Supplemental Table 1). Patients who survived had a lower MELD score (23.3 vs. 28.9, $P<0.001)$, Child score (8.73 vs. $9.71, P<0.001)$, INR (2.49 vs. $2.9, P=0.025)$, creatinine $(67.25 \mu \mathrm{mol} / \mathrm{L}$ vs. $84.95 \mu \mathrm{mol} / \mathrm{L}, P<0.001)$, TB $(270.38 \mu \mathrm{mol} / \mathrm{L}$ vs. $330.89 \mu \mathrm{mol} / \mathrm{L}, P<0.001)$, alkaline phosphatase (144.6 U/L vs. $166.68 \mathrm{U} / \mathrm{L}, P=0.002)$ value and higher albumin $(31.43 \mathrm{~g} / \mathrm{L}$ vs. $29.94 \mathrm{~g} / \mathrm{L}, P=0.032)$, haemoglobin (127.31 g/L vs. $121.62 \mathrm{~g} / \mathrm{L}, P=0.042)$ and serum sodium $(137.18 \mathrm{mmol} / \mathrm{L}$ vs. $134.72 \mathrm{mmol} / \mathrm{L}$, $P<0.001$ ) value (Table 1). Moreover, the death group had a significantly higher incidence of HE, LC, SBP, ascites when compared with the survival group $(P<0.001$ for all, Table 1).

\section{Factors associated with overall survival}

At a mean follow-up of $36.2 \pm 12.3$ months, OS was $60.8 \%$. Several factors were associated with a worse OS on univariate analysis. Specifically, increasing age (hazard ratio $(\mathrm{HR})=1.045,95 \% \mathrm{CI}$ (confidence interval) 1.030-1.060; $P<0.001)$ and $\mathrm{HE}(\mathrm{HR}=4.610,95 \%$ CI 3.125-6.799; $P<0.001)$ were associated with worse OS. Other risk factors for worse prognosis included LC $(\mathrm{HR}=2.930,95 \%$ CI 1.963-4.373; $P<0.001)$, SBP $(\mathrm{HR}=2.292,95 \%$ CI $1.573-3.340 ; P<0.001)$, ascites $(\mathrm{HR}=2.043,95 \%$ CI 1.357-3.077; $P<0.001)$, serum sodium $(\mathrm{HR}=1.000,95 \%$ CI $1.000-1.001 ; P=0.030)$, AST $(\mathrm{HR}=4.610,95 \%$ CI 3.125-6.799; $P<0.001)$, alkaline phosphatase $(\mathrm{HR}=1.002,95 \%$ CI 1.001-1.003; $P<0.001)$, TB $(\mathrm{HR}=1.002,95 \%$ CI $1.001-1.003$; $P<0.001)$, creatinine $(\mathrm{HR}=1.008,95 \%$ CI $1.005-$ $1.012 ; P<0.001)$, INR $(\mathrm{HR}=1.089,95 \%$ CI $1.019-$ $1.163 ; P=0.012$ ) (Table 2). On multivariable analysis, after adjusting for all competing risk factors, older age $(\mathrm{HR}=1.035,95 \%$ CI 1.016-1.055; $P<0.001)$, $\mathrm{HE}(\mathrm{HR}=3.688,95 \%$ CI 2.371-5.736; $P<0.001)$, alkaline phosphatase $(\mathrm{HR}=1.008,95 \%$ CI 1.004-1.011; $P<0.001)$, TB $(\mathrm{HR}=1.002,95 \%$ CI 1.001-1.003; $P=0.002)$, creatinine $(\mathrm{HR}=1.007,95 \%$ CI $1.001-$ $1.013 ; P=0.015)$, INR $(\mathrm{HR}=1.114,95 \%$ CI $1.013-$ $1.225 ; P=0.026)$ were all independently associated with decreased OS (Table 2).

\section{Comparision of overall and conditional survival}

When stratified over time, the hazard of death peaked at 2 weeks after diagnosis established and subsequently decreased thereafter (Figure 1). Actual OS at 4 weeks was $71.8 \%$ and decreased to $63.7 \%$ at 12 weeks. The 4-week CSE at 4 weeks $\left(\mathrm{CS}_{4}\right)$, which means the probability of surviving to 8 weeks after having already survival to week 4 after the date of diagnosis established, 
Table 1: Characteristics of patients with acute-on-chronic hepatitis B liver failure included in this study, stratified by different outcomes

\begin{tabular}{|c|c|c|c|}
\hline Variable & $\begin{array}{l}\text { Survival group } \\
\quad(n=169)\end{array}$ & $\begin{array}{l}\text { Death group } \\
\quad(n=109)\end{array}$ & $P$-value \\
\hline \multicolumn{4}{|l|}{ Clinical parameters } \\
\hline Age (years) & $42.04 \pm 12.29$ & $51.63 \pm 12.4$ & $<0.001$ \\
\hline Male gender No.(\%) ${ }^{*}$ & $133(78.7 \%)$ & $82(75.2 \%)$ & 0.500 \\
\hline Hepatic encephalopathy No.(\%) ${ }^{*}$ & $12(7.1 \%)$ & $45(41.3 \%)$ & $<0.001$ \\
\hline Liver cirrhosis No. $(\%)^{*}$ & $54(32.0 \%)$ & $73(67.0 \%)$ & $<0.001$ \\
\hline Infection No. $(\%)^{*}$ & $45(26.6 \%)$ & $56(51.4 \%)$ & $<0.001$ \\
\hline Ascites No.(\%) & $80(47.3 \%)$ & 76 (79.7\%) & $<0.001$ \\
\hline Hepatorenal syndrome No.(\%) & $7(4.1 \%)$ & $8(7.3 \%)$ & 0.249 \\
\hline \multicolumn{4}{|l|}{ Laboratory parameters } \\
\hline White blood cell $\left(10^{12} / \mathrm{L}\right)$ & $7.35 \pm 5.01$ & $7.41 \pm 4.05$ & 0.910 \\
\hline Haemoglobin (g/L) & $127.31 \pm 21.96$ & $121.62 \pm 23.07$ & 0.042 \\
\hline Platelet $\left(10^{9} / \mathrm{L}\right)$ & $45.27 \pm 31.07$ & $47.39 \pm 28.99$ & 0.569 \\
\hline Serum sodium (mmol/L) & $137.18 \pm 4.89$ & $134.72 \pm 5.67$ & $<0.001$ \\
\hline ALT (U/L) & $624.21 \pm 659.74$ & $540.76 \pm 628.16$ & 0.295 \\
\hline AST (U/L) & $483.76 \pm 536.35$ & $566.26 \pm 834.14$ & 0.316 \\
\hline Albumin (g/L) & $31.43 \pm 5.89$ & $29.94 \pm 5.43$ & 0.032 \\
\hline Alkaline phosphatase (U/L) & $144.6 \pm 47.03$ & $166.68 \pm 71.62$ & 0.002 \\
\hline Total bilirubin $(\mu \mathrm{mol} / \mathrm{L})$ & $270.38 \pm 153.14$ & $330.89 \pm 170.34$ & 0.002 \\
\hline Creatinine $(\mu \mathrm{mol} / \mathrm{L})$ & $67.25 \pm 26.46$ & $84.95 \pm 56.55$ & $<0.001$ \\
\hline INR & $2.49 \pm 1.64$ & $2.9 \pm 1.22$ & 0.025 \\
\hline \multicolumn{4}{|l|}{ Scoring systems } \\
\hline MELD score & $23.3 \pm 6.39$ & $28.9 \pm 8.98$ & $<0.001$ \\
\hline Child score & $8.73 \pm 1.58$ & $9.71 \pm 1.4$ & $<0.001$ \\
\hline
\end{tabular}

NOTE: ALT, alanine aminotranferase; AST, aspartate aminotranferase; INR, international normalized ratio; MELD, model for end-stage liver disease.

"Dichotomous values.

was $89.5 \%$. Similarly, the 8 -week $\mathrm{CS}_{4}$, which means the probability of surviving to 12 weeks after having already survival to week 8 , was $94.6 \%$ compared with an actual OS 12-week rate of $63.7 \%$. 12-week $\mathrm{CS}_{4}$ rates increased over from $76.2 \%$ to $97.7 \%(P<0.001)$, whereas actual OS deceased over time from $71.8 \%$ at 4 weeks to $63.7 \%$ at 16 weeks $(P<0.001)$. CSE based on time already survived are summarized in Table 3 . The probability of surviving an additional 4 weeks, given that the patient had survived for $1,3,5,7,9$ weeks was $74 \%, 86 \%, 92 \%, 93 \%, 97 \%$, respectively.

As shown in the Kaplan-Meier analysis, OS worsened with increasing MELD score and Child grade $\mathrm{C}$ (both $\log$-rank $P<0.001$, Figure 2A and 2C). For example, 8 -week actual OS for patients with MELD $\leq 25$ was
$81 \%$ and decreased with worsening extent of disease: $52 \%$ for MELD $>25$. The calculated $\mathrm{CS}_{4}$ exceeded the actual OS for all corresponding MELD score (Figure 2B). Furthermore, this difference was more significant for those patients who were initially predicted to have poorer prognosis. For example, patients with MELD $>25$ had a $\mathrm{CS}_{4}$ of $92 \%$ at 8 weeks compared with an actual OS of $51 \%$ at 12 weeks $(\Delta 41 \%)$. Similarly, patients with Child grade $\mathrm{C}$ had a $\mathrm{CS}_{4}$ of $92 \%$ at 8 weeks compared with an actual OS of $56 \%$ at 12 weeks $(\Delta 36 \%)$ (Figure 2D). Conversely, patients with "favorable" characteristics had smaller differences between actual OS and CSE. Specifically, patients with MELD $\leq 25$ had an actual 12-week OS of $78 \%$ compared to a 8 -week $\mathrm{CS}_{4}$ of $96 \%$ $(\Delta 18 \%)$. Similarly, patients with Child grade A and B had 
Table 2: Univariable and multivariable Cox proportional hazards analysis for overall survival of acute-on-chronic hepatitis B liver failure

\begin{tabular}{|c|c|c|c|c|c|c|}
\hline & \multicolumn{3}{|c|}{ Univariable analysis } & \multicolumn{3}{|c|}{ Multivariable analysis } \\
\hline & HR & $95 \% \mathrm{CI}$ & $P$-value & HR & $95 \% \mathrm{CI}$ & $P$-value \\
\hline \multicolumn{7}{|l|}{ Clinical parameters } \\
\hline Age (years) & 1.045 & $1.030-1.060$ & $<0.001$ & 1.035 & $1.016-1.055$ & $<0.001$ \\
\hline Male gender* & 0.903 & $0.585-1.390$ & 0.647 & 1.241 & $0.744-2.069$ & 0.407 \\
\hline Hepatic encephalopathy* & 4.610 & $3.125-6.799$ & $<0.001$ & 3.688 & $2.371-5.736$ & $<0.001$ \\
\hline Liver cirrhosis ${ }^{*}$ & 2.930 & $1.963-4.373$ & $<0.001$ & 1.644 & $0.948-2.852$ & 0.077 \\
\hline Infection $^{*}$ & 2.292 & $1.573-3.340$ & $<0.001$ & 1.370 & $0.885-2.12$ & 0.158 \\
\hline Ascites $^{*}$ & 2.043 & $1.357-3.077$ & $<0.001$ & 1.102 & $0.645-1.884$ & 0.722 \\
\hline Hepatorenal syndrome* & 1.834 & $0.893-3.770$ & 0.099 & 1.125 & $0.435-2.906$ & 0.808 \\
\hline \multicolumn{7}{|l|}{ Laboratory parameters } \\
\hline White blood cell $\left(10^{12} / \mathrm{L}\right)$ & 1.008 & $0.972-1.046$ & 0.666 & 0.962 & $0.904-1.023$ & 0.216 \\
\hline Haemoglobin $(\mathrm{g} / \mathrm{L})$ & 0.991 & $0.983-1.000$ & 0.430 & 1.005 & $0.994-1.017$ & 0.376 \\
\hline Platelet $\left(10^{9} / \mathrm{L}\right)$ & 1.001 & $0.995-1.007$ & 0.761 & 1.000 & $0.993-1.007$ & 0.970 \\
\hline Serum sodium (mmol/L) & 0.936 & $0.908-0.965$ & $<0.001$ & 0.963 & $0.927-1.001$ & 0.058 \\
\hline ALT (U/L) & 1.000 & $1.000-1.000$ & 0.404 & 1.000 & $0.999-1.000$ & 0.155 \\
\hline AST (U/L) & 1.000 & $1.000-1.001$ & 0.030 & 1.000 & $1.000-1.001$ & 0.086 \\
\hline Albumin $(\mathrm{g} / \mathrm{L})$ & 0.969 & $0.938-1.000$ & 0.053 & 1.036 & $0.988-1.087$ & 0.146 \\
\hline Alkaline phosphatase (U/L) & 1.002 & $1.001-1.003$ & $<0.001$ & 1.008 & $1.004-1.011$ & $<0.001$ \\
\hline Total bilirubin $(\mu \mathrm{mol} / \mathrm{L})$ & 1.002 & $1.001-1.003$ & $<0.001$ & 1.002 & $1.001-1.003$ & 0.002 \\
\hline Creatinine $(\mu \mathrm{mol} / \mathrm{L})$ & 1.008 & $1.005-1.012$ & $<0.001$ & 1.007 & $1.001-1.013$ & 0.015 \\
\hline INR & 1.089 & $1.019-1.163$ & 0.012 & 1.114 & $1.013-1.225$ & 0.026 \\
\hline
\end{tabular}

NOTE: ALT, alanine aminotranferase; AST, aspartate aminotranferase; INR, international normalized ratio; MELD, model for end-stage liver disease; HR, hazard ratio; CI, confidence interval.

*Dichotomous values.

a 8 -week $\mathrm{CS}_{4}$ of $96 \%$ compared with an actual OS of $74 \%$ at 12 weeks $(\Delta 22 \%)$.

$\mathrm{CS}_{4}$ also was stratified by different clinical and laboratory variables of known prognostic importance, such as age, $\mathrm{HE}$ and INR. $\mathrm{CS}_{4}$ increased over time among patients younger than 45 years old $(83 \%-98 \%, \Delta 15 \%)$ and older than 45 years $(68 \%-97 \%, \Delta 29 \% ; P<0.001)$ (Figure 3A, 3B). The differences in 8-week $\mathrm{CS}_{4}$ over time were more pronounced among patients with worse prognostic features. Smaller changes over time in 8-week $\mathrm{CS}_{4}$ were seen in patients without HE $(85 \%-97 \%, \Delta 12 \%)$ compared with patients with HE $(40 \%-100 \%, \Delta 60 \%$; $P<0.001$ ) (Figure 3C, 3D). Similarly, patients with INR $>2.5(64 \%-97 \%, \Delta 33 \%)$ had larger difference in 8-week $\mathrm{CS}_{4}$ compared with patients with INR $\leq 2.5$ $(86 \%-98 \%, \Delta 12 \%)$ (Figure 3E, 3F). These patterns were also observed in 8 -week $\mathrm{CS}_{4}$ differences between patients with MELD $\leq 25(89 \%-98 \%, \Delta 9 \%)$ and MELD > $25(61 \%-97 \%, \Delta 36 \%)$, as well as between patients with Child grade A and B $(82 \%-98 \%, \Delta 16 \%)$ and Child grade C $(69 \%-97 \%, \Delta 28 \%)$ (all $P<0.001)$. Similarity, same trends had been found in the remaining prognostic factors (TB, creatinine, alkaline phosphatase; Figure 4A-4F).

\section{DISCUSSION}

ACHBLF is an aggressive critical condition with high short-term mortality risk $[1,5,25]$. There is still no easy-to-use mortality risk prediction tool derived from a population-based prospective cohort study [4]. To our knowledge, this study is the first to provide strong support for the use of CSE in the prediction of short term mortality in ACHBLF patients as these estimates have a stronger 

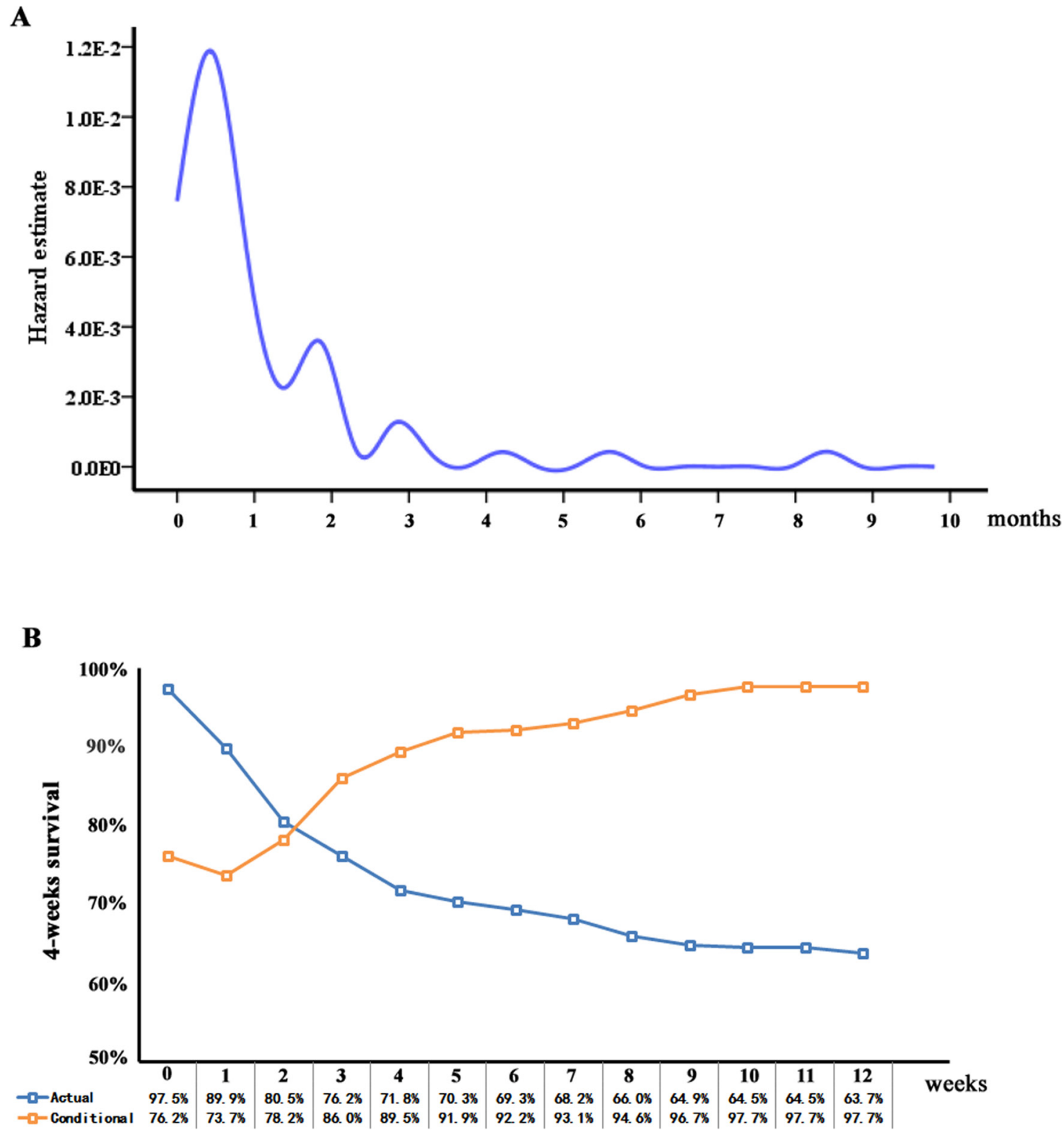

Figure 1: A. Hazard estimate of death in this large multicenter cohort and B. 4-week conditional survival estimate relative to actual survival.

dynamic predictive ability compared with traditional OS. With wide-ranging survival estimates, static prediction of OS based on data around the time of diagnosis may not be accurate as patients had survived for a period of time.

In this large prospective multicenter study, the actual OS at $2,4,6,8,12$ weeks were $80.5 \%, 71.8 \%, 69.3 \%$, $66.0 \%$ and $63.7 \%$, respectively. However, we had also noted that the hazard of death did not remain constant over time. Rather, the probability of CSE increased over time based on survival time already accumulated, providing support for the concept that CSE may be a better estimate of prognosis as survival time accrues. In fact, on average, CSE increased over time and were significantly higher than actual OS, which was consistent with the trends from other research groups [13-14, 17]. Furthermore, the magnitude in difference between CSE and OS were highest among patients with worse prognostic features.

Depending on the patient and liver-specific factors, including age, HE, INR, MELD, Child score, CSE may provide a more accurate and clinically relevant patientspecific survival estimate. However, these differences were not uniform across all patients. Patients with worse prognostic features had a higher increase in CSE based on actual time survived. For example, patients without HE showed only a $12 \%$ increase in 8 -week $\mathrm{CS}_{4}$ compared with a $60 \%$ increase in patients with $\mathrm{HE}$ and the critical role of 
Table 3: Proportion of patients with acute-on-chronic hepatitis B liver failure who reach a certain survival time point, given that they have already survived a certain amount of time

\begin{tabular}{|c|c|c|c|c|c|c|c|c|c|c|c|c|}
\hline \multirow{2}{*}{$\begin{array}{l}\text { Total } \\
\text { survival } \\
\text { time }\end{array}$} & \multicolumn{12}{|c|}{ If the patient has survived to } \\
\hline & \multicolumn{12}{|c|}{1 week 2 weeks 3 weeks 4 weeks 5 weeks 6 weeks 7 weeks 8 weeks 9 weeks 10 weeks 11 weeks 12 weeks } \\
\hline \multicolumn{13}{|l|}{1 week } \\
\hline 2 weeks & $92.2 \%$ & & & & & & & & & & & \\
\hline 3 weeks & $82.6 \%$ & $89.5 \%$ & & & & & & & & & & \\
\hline 4 weeks & $78.1 \%$ & $84.7 \%$ & $94.6 \%$ & & & & & & & & & \\
\hline 5 weeks & $73.7 \%$ & $79.9 \%$ & $89.2 \%$ & $94.3 \%$ & & & & & & & & \\
\hline 6 weeks & $72.2 \%$ & $78.2 \%$ & $87.4 \%$ & $92.4 \%$ & $98.0 \%$ & & & & & & & \\
\hline 7 weeks & $71.0 \%$ & $77.0 \%$ & $86.0 \%$ & $90.9 \%$ & $96.5 \%$ & $98.4 \%$ & & & & & & \\
\hline 8 weeks & $69.9 \%$ & $75.8 \%$ & $84.7 \%$ & $89.5 \%$ & $94.9 \%$ & $96.9 \%$ & $98.4 \%$ & & & & & \\
\hline 9 weeks & $67.7 \%$ & $73.4 \%$ & $81.9 \%$ & $86.6 \%$ & $91.9 \%$ & $93.8 \%$ & $95.2 \%$ & $96.8 \%$ & & & & \\
\hline 10 weeks & $66.5 \%$ & $72.1 \%$ & $80.6 \%$ & $85.2 \%$ & $90.3 \%$ & $92.2 \%$ & $93.7 \%$ & $95.2 \%$ & $98.3 \%$ & & & \\
\hline 11 weeks & $66.2 \%$ & $71.7 \%$ & $80.1 \%$ & $84.7 \%$ & $89.8 \%$ & $91.7 \%$ & $93.1 \%$ & $94.6 \%$ & $97.8 \%$ & $99.4 \%$ & & \\
\hline 12 weeks & $66.2 \%$ & $71.7 \%$ & $80.1 \%$ & $84.7 \%$ & $89.8 \%$ & $91.7 \%$ & $93.1 \%$ & $94.6 \%$ & $97.8 \%$ & $99.4 \%$ & $100.0 \%$ & \\
\hline 13 weeks & $65.4 \%$ & $70.9 \%$ & $79.2 \%$ & $83.7 \%$ & $88.8 \%$ & $90.6 \%$ & $92.1 \%$ & $93.5 \%$ & $96.7 \%$ & $98.3 \%$ & $98.9 \%$ & $98.9 \%$ \\
\hline
\end{tabular}

A
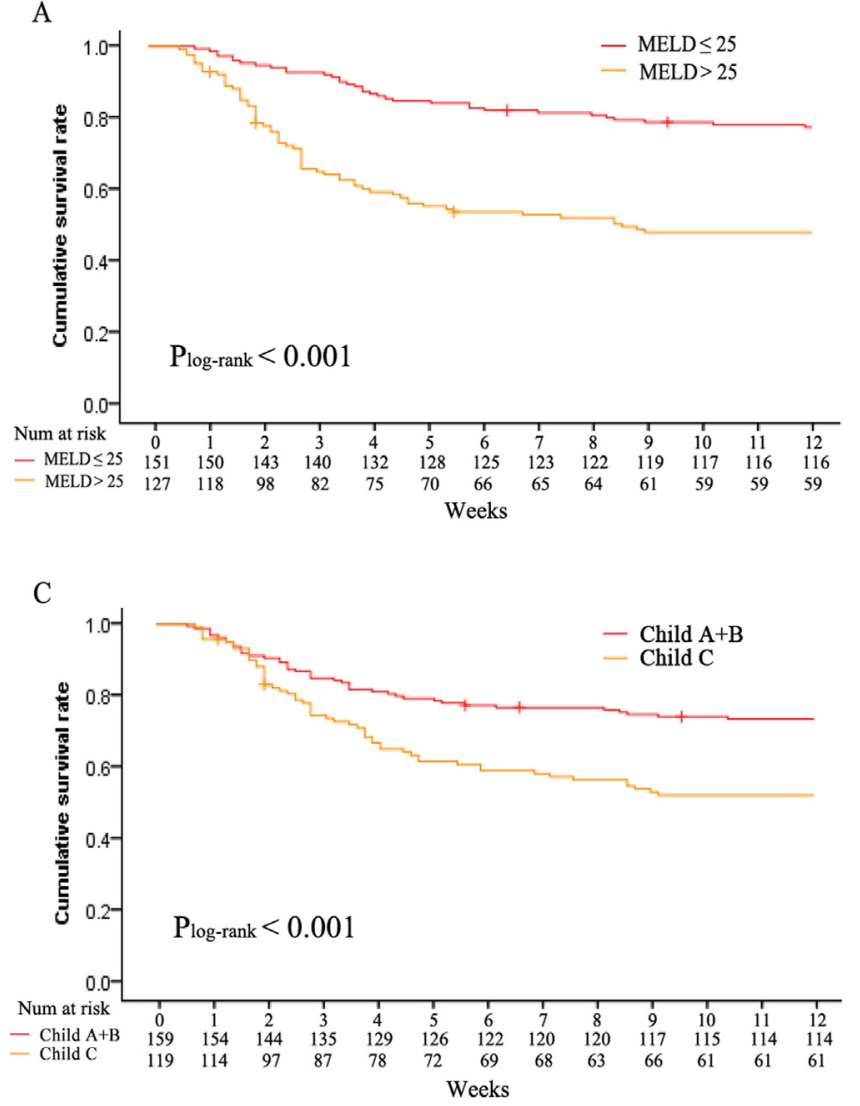

B

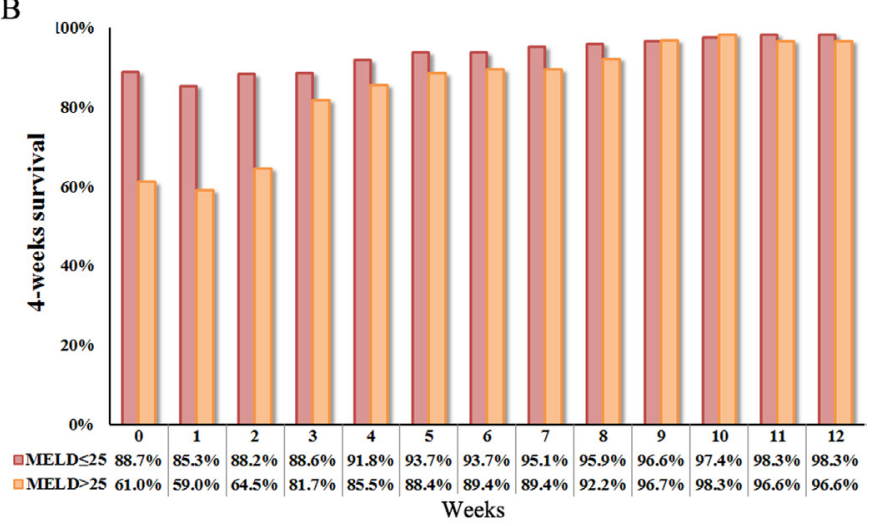

D

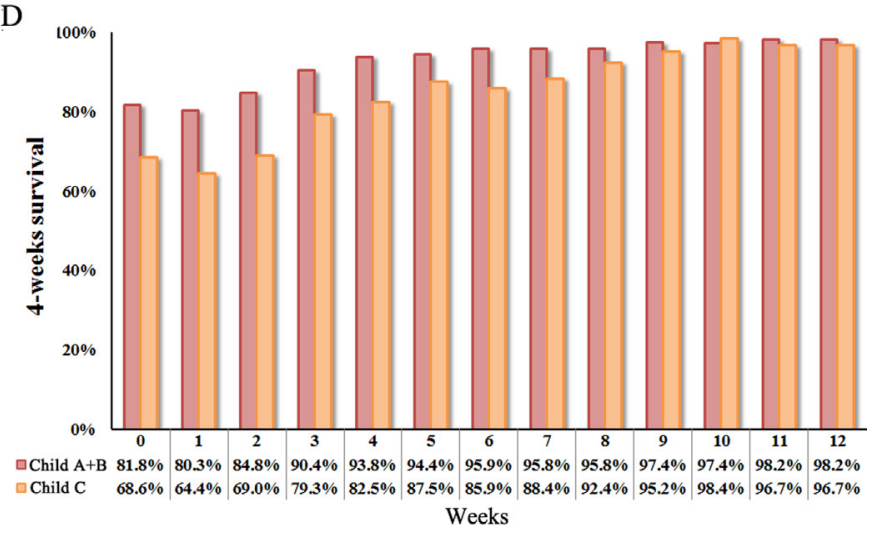

Figure 2: Overall survival stratified by A. MELD score (log-rank $P<0.001)$, C. Child score $(\log -\operatorname{rank} P<0.001)$ and conditional survival estimates stratified by $\mathbf{B}$. MELD score and $\mathbf{D}$. Child score. 
A
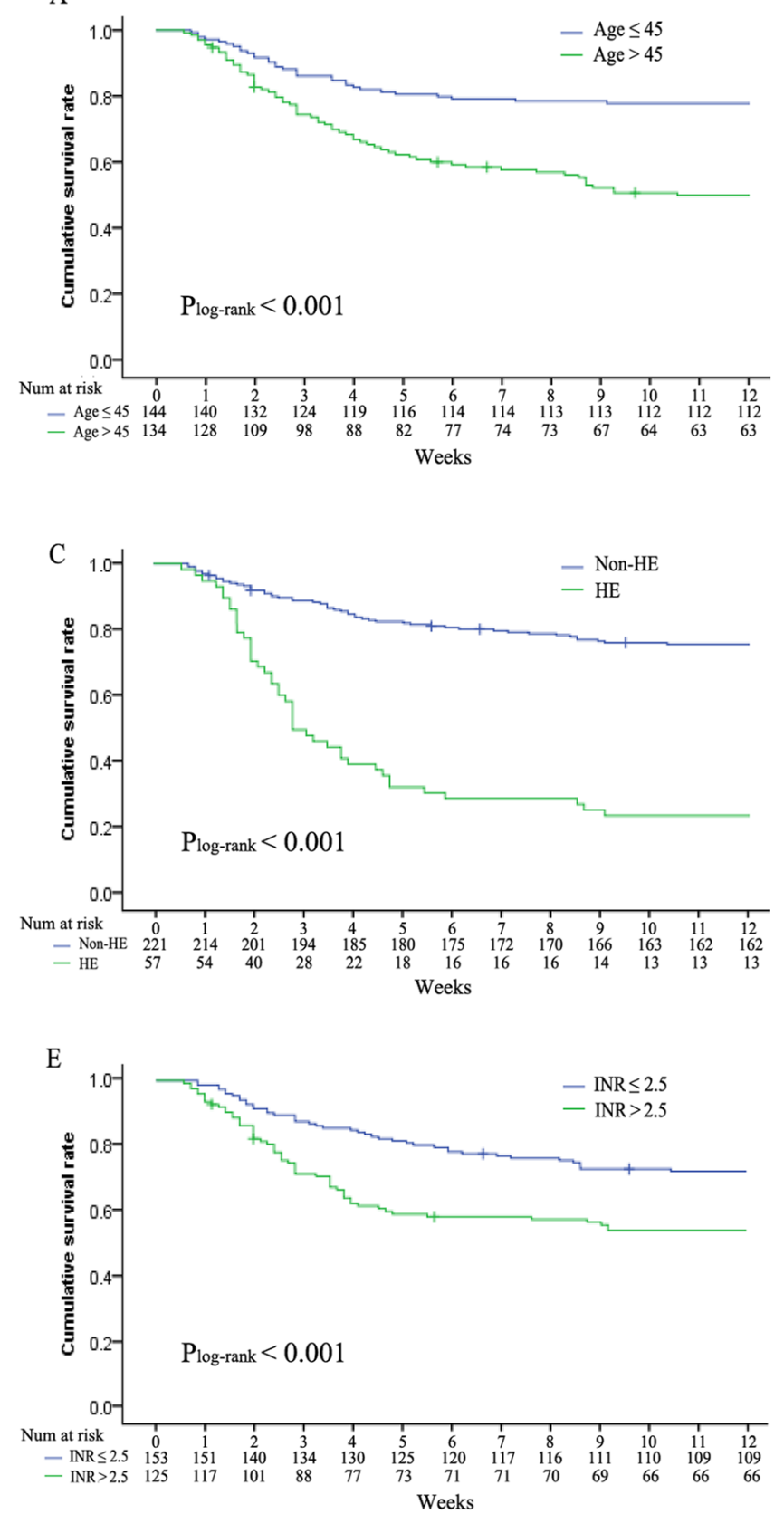

B
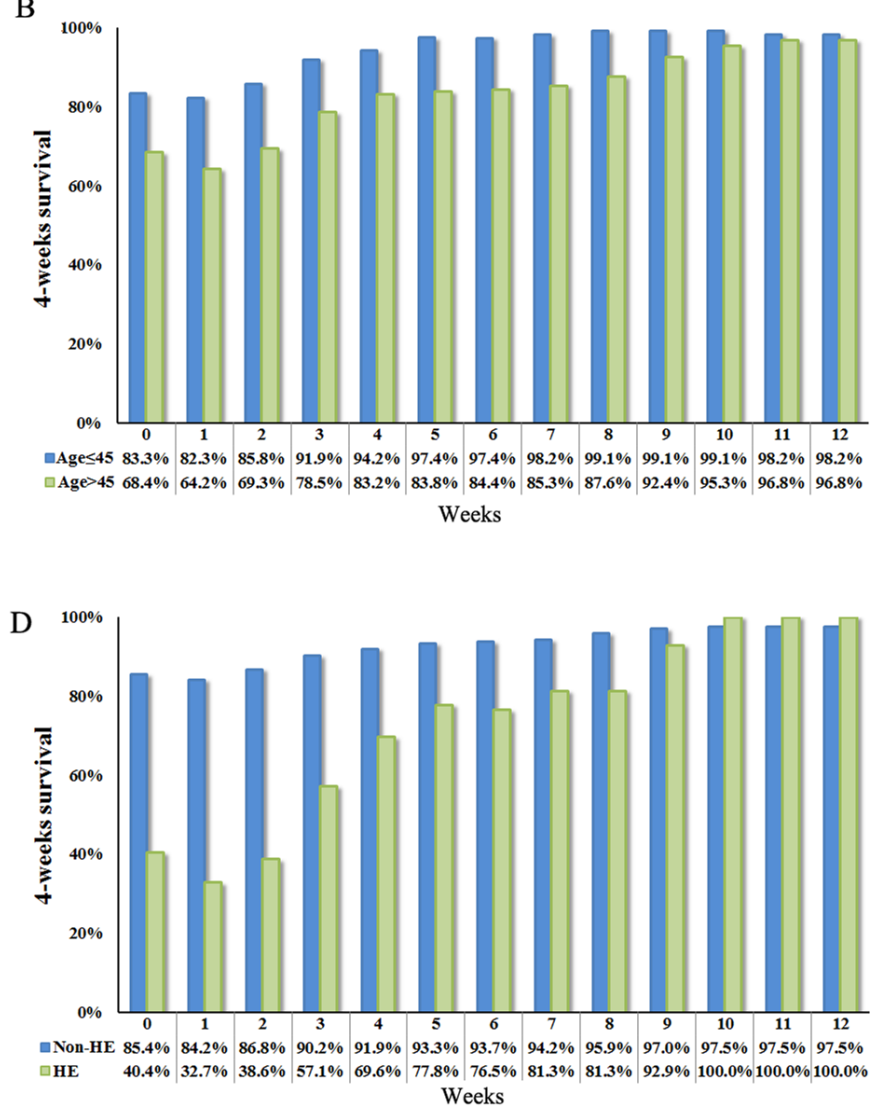

Figure 3: Overall survival stratified by A. age (log-rank $P<0.001)$, C. hepatic encephalopathy (log-rank $P<0.001)$, E. international normalized ratio $(\log$-rank $P<0.001)$ and conditional survival estimates stratified by $\mathbf{B}$. age, $\mathbf{D}$. hepatic encephalopathy and $\mathbf{F}$. international normalized ratio.

HE in ACHBLF has been recently been highlighted [26]. Similarly, patients with MELD > 25 showed a $36 \%$ increase in 8 -week $\mathrm{CS}_{4}$ compared with a $10 \%$ increase in patients with MELD $\leq 25$. As such, CSE should be favored and used in this instance, particularly in high-risk patients.

Compared with existing scoring systems that we had described, including CLIF Consortium Organ Failure score (CLIF-C OFs), CLIF-SOFA, ANN, ALPH-Q, LRM, MELD et al. [7-11, 27], current CSE do not require physicians to perform complex calculations, but rather, they can easily extract the estimated risk and the impact on risk when various risk factors are added or removed. Furthermore, the CSE enable physicians to more easily engage with a target patient in an individual discussion of risk and thus enhance risk communication [28].

There are, however, some limitations of this study. First, because the present study is a multicenter analysis, there may have been selection bias in the cohort. However, 
A

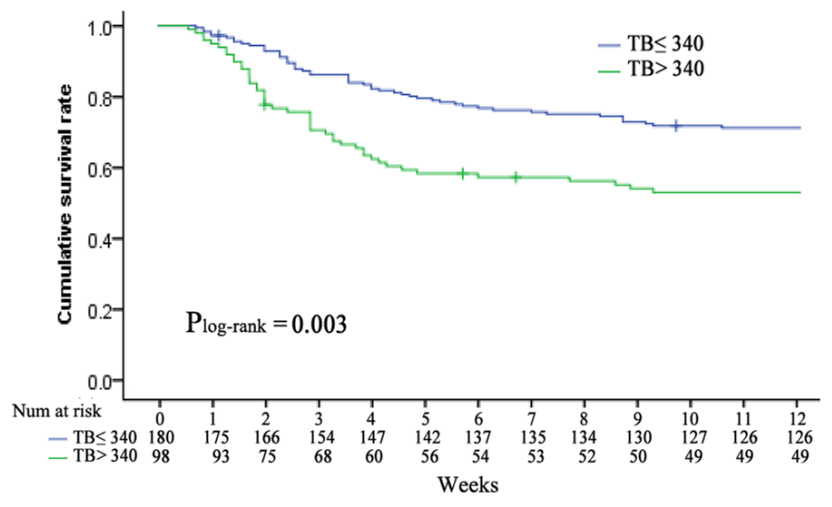

C

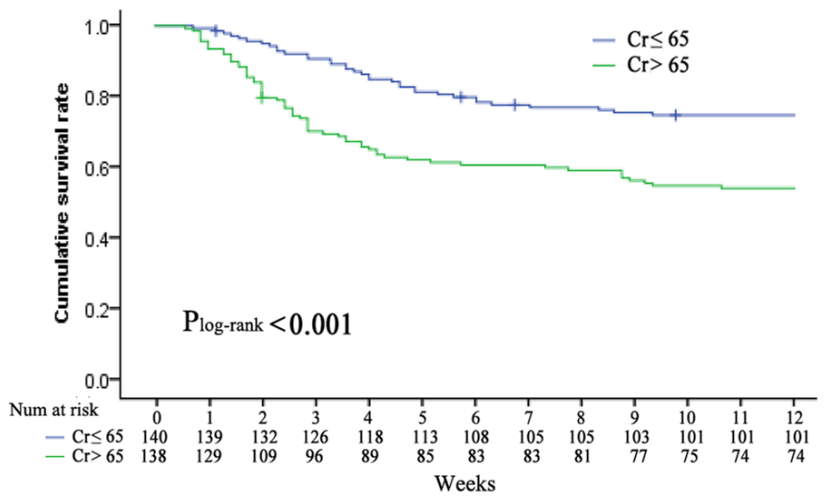

E

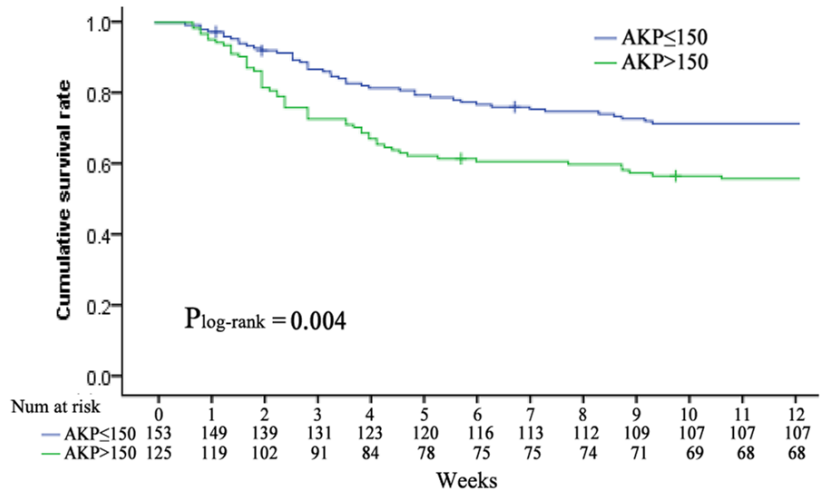

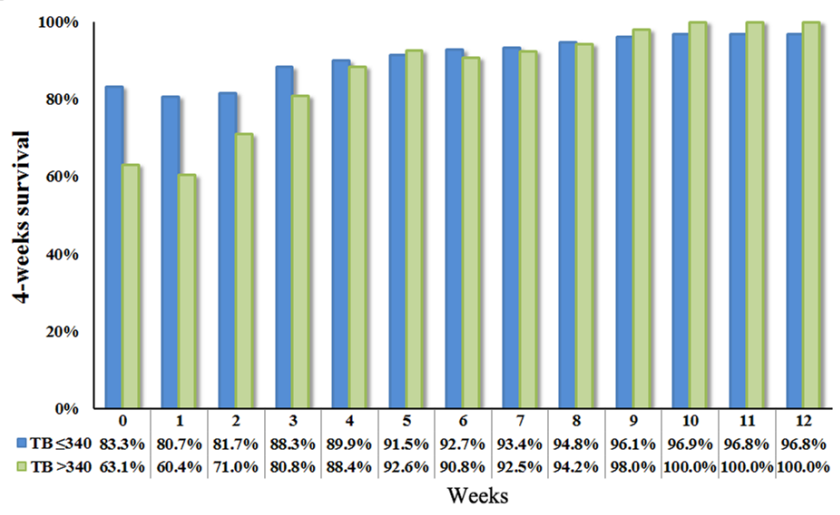

D

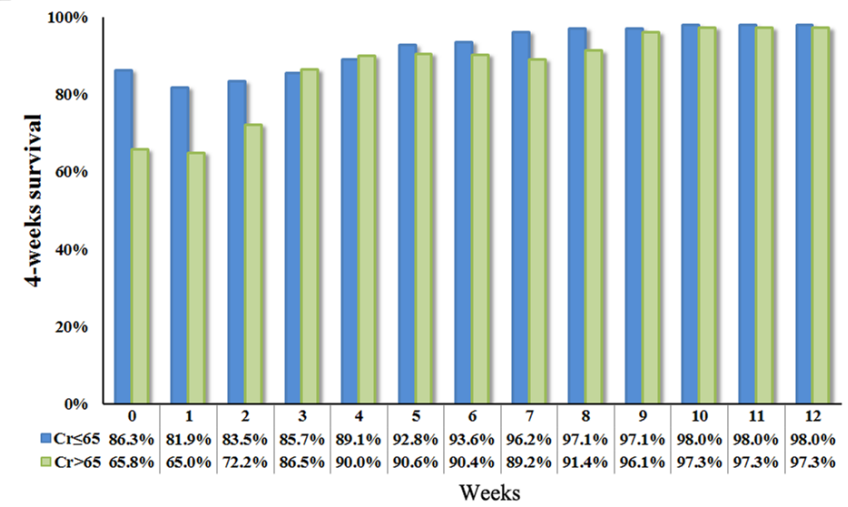

F

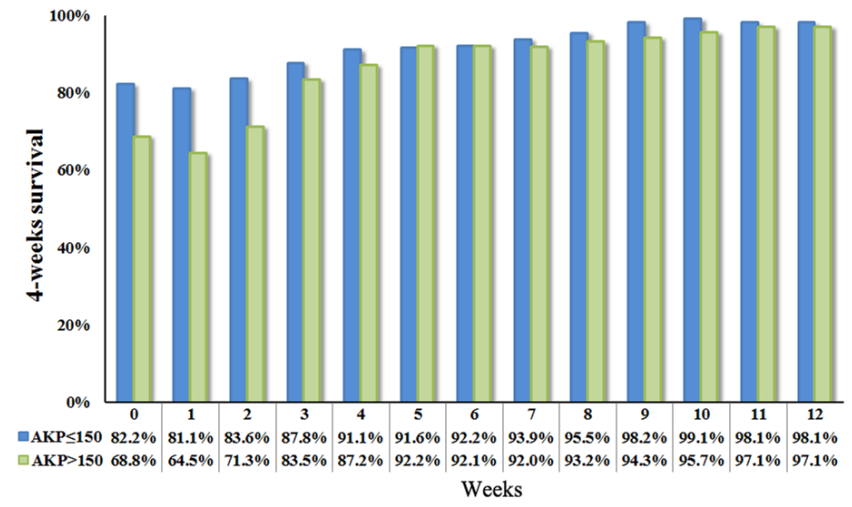

Figure 4: Overall survival stratified by A. total bilirubin (log-rank $P=0.003)$, C. creatinine $(\log$-rank $P<0.001)$, E. alkaline phosphatase $(\log$-rank $P<0.004)$ and conditional survival estimates stratified by B. total bilirubin, D. creatinine and F. alkaline $\mathrm{p}$ hosphatase.

the multicenter nature of the current study indeed provides support to the generalizability of our results. Second, current CSE only rendered the data of the first 3 months. As a critical disease, death was not uncommon in the early stages and paying more attention to short-term mortality will allow a better future assessment of long-term survival. Finally, external validation of this CSE in a more diverse patient population, preferentially in the clinical setting, is necessary. In summary, this easy-to-use CSE should permit physicians to assess the individual risk of ACHBLF patients and facilitate risk communications between physicians and patients. 


\section{MATERIALS AND METHODS}

\section{Study population}

We prospectively enrolled patients from three separate medical centers (the First Affiliated Hospital of Wenzhou Medical University, Ningbo No. 2 Hospital and Tianjin Infectious Disease Hospital, from December 2010 to December 2013) with the same medical record systems. The start date of the follow-up was the date of diagnosis of ACHBLF. All patients were followed up for at least 3 months. Written informed consent was obtained from each patient included in the study and the research protocol of the study was approved by the Ethics Committee of the First Affiliated Hospital of Wenzhou Medical University, Ningbo No. 2 Hospital and Tianjin Infectious Disease Hospital.

\section{Inclusion and exclusion criteria}

ACLF was diagnosed according to the recommendation of the APASL [2]. In brief, ACLF is defined as acute hepatic insult manifesting as jaundice and coagulopathy, complicated within 4 weeks by ascites or encephalopathy in a patient with previously diagnosed or undiagnosed chronic liver disease. ACHBLF is defined as ACLF caused solely by HBV. Patients who meet the following criteria were excluded: 1) infected and/or co-infected with non-HBV; 2) autoimmune diseases; 3) alcohol abuse; 4) past or current HCC; 5) toxic caused liver disease; 6) pregnancy; 7) liver transplantation previously. Once diagnosis of ACHBLF, the cares that provided to the included patients at three centers were same, and in accordance with the Asia-Pacific consensus recommendations [2]. This routinely included oral antiviral agents, absolute bed rest, energy supplements and vitamins, intravenous drop infusion albumin, maintenance water, electrolyte and acid-base equilibrium and prevention and treatment complications, etc.

\section{Data collection}

A detailed history of all the patients was taken when they were in hospital. Patient medical history was recorded upon admission and every 2-weeks during follow-up. Patient characteristics were detected within the first 24 hours after the established diagnosis of ACHBLF. Confirmatory physical examination, laboratory tests and abdominal ultrasound scanning were performed.

Clinical parameters included age, gender, body mass index, blood pressure, hepatic encephalopathy (HE), liver cirrhosis (LC), ascites and hepatorenal syndrome (HRS). The HE grade was re-classified into 0: non-HE, 1: mild (grade 1-2) and 2: severe (grade 3-4) according to West-Haven criteria [29]. LC was defined by the following combined parameters: (1) a score greater than 2 according to the aspartate aminotransferase (AST) to platelet ratio using the formula: [AST/upper limit of normal]/platelet count $\left(\times 10^{9} / \mathrm{L}\right) \times 100$ [30], (2) ultrasonographic evidence of a small sized liver with and without splenomegaly/ ascites, and (3) an albumin level less than $35 \mathrm{~g} / \mathrm{L}$ without other identifiable causes of hypoalbuminemia such as renal loss or gastrointestinal loss. The detection of ascites included history, physical examination, abdominal ultrasound, and laboratory assessment of liver function, renal function, serum and urine electrolytes. We re-classified ascites grade into 0 : non-ascites, 1 : mild (grade 1), 2: moderate to severe (grade 2-3) [31]. HRS was defined as low glomerular filtration rate, as indicated by serum creatinine of $>1.5 \mathrm{mg} / \mathrm{dL}$ or $24-\mathrm{h}$ creatinine clearance $<40 \mathrm{ml} / \mathrm{min}$, without the presence of chronic kidney diseases [32].

Laboratory parameters including alanine aminotransferase, AST, total bilirubin (TB), albumin, platelet count, hemoglobin, serum creatinine, international normalized ratio (INR), serum sodium and potassium. HBV serologic markers were collected for each patient (Abbott, AXSYM). Serum HBV DNA was measured by quantitative PCR assay (Roche Amplicor, limit of detectability of $100 \mathrm{IU} / \mathrm{ml}$ ) after admission. Hepatitis $\mathrm{C}$ virus antibody and human immunodeficiency virus antibody were detected using ELISA (IEGAN, Freedom evolyzer/150). Antinuclear antibody was evaluated using indirect immunofluorescence and soluble liver antigen/ liver pancreas antigen, anti-liver/kidney microsomal antibody Type 1 and anti- liver cytosol antibody Type 1 were evaluated using immunoblot analysis (Euroimmun, Lubeck, Germany).

\section{Scoring systems and prognostic models}

Child score, which included HE, PT, ascites, TB and serum albumin, was assessed according to the standard criteria [33]. MELD score were calculated according to the Malinchoc formula: $\mathrm{R}=9.57 \times \ln ($ creatinine $[\mathrm{mg} / \mathrm{dL}])$ $+3.78 \times \ln ($ bilirubin $[\mathrm{mg} / \mathrm{dL}])+11.2 \times \ln (\mathrm{INR})+6.43 \times$ (aetiology: 0 if cholestatic or alcoholic, 1 otherwise) [27].

\section{Follow-up}

Patients were followed up for every 2 weeks for 3 months when the diagnosis was established, every 1 month thereafter for a total of 1 year and every 3 months thereafter. Information on death was obtained either from the patient's social security death index, outpatient medical records, or notifications from the family of the deceased. The deadline of follow-up time was March 1, 2015.

\section{Statistical analysis}

The Kolmogorov-Smirnov test was applied to determine whether sample data were likely to be derived 
from a normal distribution population. Continuous variables of normal and skewed distribution are expressed as mean \pm standard deviation and median (interquartile range), respectively. Categorical values were expressed by absolute and relative frequencies. Differences in variables were analyzed using Student $t$-tests (for normally distributed data) or Wilcoxon's Sign Rank Test (for skewed distributed data). The Chi-square test or the Fisher's exact test was used for categorical data as appropriate. OS estimates for the entire study population were generated using the Kaplan-Meier method calculated from the date of diagnosis to the date of last follow-up or death. The association of relevant variables with OS was assessed using Cox proportional hazards models. Variables in the univariate Cox regression analysis were progressed to a multivariate analysis using forward stepwise selection. According to the previous findings [7-9], the risk of death is greatest within the first three months after the date of diagnosis established. So, 12-week was chosen as the main cutoff of timeline. CSE was calculated as the probability of survival for an additional 4 week $\left(\mathrm{CS}_{4}\right)$, given that the patient had survived for 1, 2, 3, 4, or more weeks, calculated as $\mathrm{CS}_{4}=\mathrm{OS}_{(\mathrm{X}+4)} / \mathrm{OS}_{(\mathrm{X})}$. For example, 4-week CSE among patients who have survived 2 weeks from the date of diagnosis is calculated by dividing the 6-week OS rate by the 2-week OS rate. Changes in $\mathrm{CS}_{4}$ over time were assessed using linear regression. For all analyses, a $P$ value of $<0.05$ was considered statistically significant. Statistical analysis was performed using SPSS version 20.0 (SPSS, Chicago, IL, USA).

\section{ACKNOWLEDGMENTS}

Author contributions: Zheng MH, Wu SJ, Yan HD, Li $\mathrm{H}$ and Chen YP designed the study. Shi KQ, Yan HD and Li H collected data. Zhu GQ and Xie YY did the statistical analyses. Wu SJ and Wu FL prepared figures. Zheng MH, Wu SJ and Chen YP reviewed the results, interpreted data, and wrote the manuscript. All authors have made an intellectual contribution to the manuscript and approved the submission.

\section{CONFLICTS OF INTEREST}

The authors report no declarations of interest.

\section{FUNDING INFORMATION}

This work was supported by grants from National Science and Technology Major Project of China (2012ZX10002004), Scientific Research Foundation of Wenzhou, Zhejiang Province, China (H20090014, Y20090269), Health Bureau of Zhejiang Province (2010KYB070), Research Foundation of Education Bureau of Zhejiang Province (Y201009942), Fresh
Talent Program for Science and Technology Department of Zhejiang Province (2013R413018, 2013R413035 and 2013R413015), Research Funds for Tian Qing Liver Diseases (TQGB20120057) and Project of New Century 551 Talent Nurturing in Wenzhou. Registration number: ChiCTR-PRNRC-11001172.

\section{REFERENCES}

1. Moreau R, Arroyo V. Acute on Chronic Liver Failure: A New Clinical Entity. Clin Gastroenterol Hepatol. 2015; 13:836-841.

2. Sarin SK, Kumar A, Almeida JA, Chawla YK, Fan ST, Garg H, de Silva HJ, Hamid SS, Jalan R, Komolmit P, Lau GK, Liu Q, Madan K, et al. Acute-on-chronic liver failure: consensus recommendations of the Asian Pacific Association for the study of the liver (APASL). Hepatol Int. 2009; 3:269-282.

3. Liu J, Fan D. Hepatitis B in China. Lancet. 2007; 369:1582-1583.

4. Wu FL, Shi KQ, Chen YP, Braddock M, Zou H, Zheng MH. Scoring systems predict the prognosis of acute-on-chronic hepatitis B liver failure: an evidence-based review. Expert Rev Gastroenterol Hepatol. 2014; 8:623-632.

5. Seto WK, Lai CL, Yuen MF. Acute-on-chronic liver failure in chronic hepatitis B. J Gastroenterol Hepatol. 2012; 27:662-669.

6. Siddiqui MS, Stravitz RT. Intensive care unit management of patients with liver failure. Clin Liver Dis. 2014; 18:957-978.

7. Wu SJ, Yan HD, Zheng ZX, Shi KQ, Wu FL, Xie YY, Fan YC, Ye BZ, Huang WJ, Chen YP, Zheng MH. Establishment and Validation of ALPH-Q Score to Predict Mortality Risk in Patients With Acute-on-Chronic Hepatitis B Liver Failure: A Prospective Cohort Study. Medicine (Baltimore). 2015; 94:e403.

8. Zheng MH, Shi KQ, Fan YC, Li H, Ye C, Chen QQ, Chen YP. A Model to Determine 3-Month Mortality Risk in Patients With Acute-on-Chronic Hepatitis B Liver Failure. Clin Gastroenterol Hepatol. 2011; 9:351-356. e353.

9. Zheng MH, Shi KQ, Lin XF, Xiao DD, Chen LL, Liu WY, Fan YC, Chen YP. A model to predict 3-month mortality risk of acute-on-chronic hepatitis B liver failure using artificial neural network. J Viral Hepat. 2013; 20:248-255.

10. Jalan R, Saliba F, Pavesi M, Amoros A, Moreau R, Gines P, Levesque E, Durand F, Angeli P, Caraceni P, Hopf C, Alessandria C, Rodriguez E, et al. Development and validation of a prognostic score to predict mortality in patients with acute-on-chronic liver failure. J Hepatol. 2014; 61:1038-1047.

11. Moreau R, Jalan R, Gines P, Pavesi M, Angeli P, Cordoba J, Durand F, Gustot T, Saliba F, Domenicali M, Gerbes A, Wendon J, Alessandria C, et al. Acute-on-chronic liver 
failure is a distinct syndrome that develops in patients with acute decompensation of cirrhosis. Gastroenterology. 2013; 144:1426-1437. 1437 e1421-1429.

12. Most L, Hothorn T. Conditional Transformation Models for Survivor Function Estimation. Int J Biostat. 2015.

13. Bischof DA, Kim Y, Dodson R, Jimenez MC, Behman R, Cocieru A, Fisher SB, Groeschl RT, Squires MH 3rd, Maithel SK, Blazer DG 3rd, Kooby DA, Gamblin TC, et al. Conditional Disease-Free Survival After Surgical Resection of Gastrointestinal Stromal Tumors: A Multi-institutional Analysis of 502 Patients. JAMA Surg. 2015.

14. Kim Y, Ejaz A, Spolverato G, Squires MH, Poultsides G, Fields RC, Bloomston M, Weber SM, Votanopoulos K, Acher AW, Jin LX, Hawkins WG, Schmidt C, et al. Conditional survival after surgical resection of gastric cancer: a multi-institutional analysis of the us gastric cancer collaborative. Ann Surg Oncol. 2015; 22:557-564.

15. Dikken JL, Baser RE, Gonen M, Kattan MW, Shah MA, Verheij M, van de Velde CJ, Brennan MF, Coit DG. Conditional probability of survival nomogram for 1-, 2-, and 3-year survivors after an R0 resection for gastric cancer. Ann Surg Oncol. 2013; 20:1623-1630.

16. Sun M, Trinh QD, Karakiewicz PI. Conditional survival of patients with metastatic renal-cell carcinoma. Lancet Oncol. 2012; 13:e462.

17. Harshman LC, Xie W, Bjarnason GA, Knox JJ, MacKenzie M, Wood L, Srinivas S, Vaishampayan UN, Tan MH, Rha SY, Donskov F, Agarwal N, Kollmannsberger C, et al. Conditional survival of patients with metastatic renal-cell carcinoma treated with VEGF-targeted therapy: a population-based study. Lancet Oncol. 2012; 13:927-935.

18. Skuladottir H, Olsen JH. Conditional survival of patients with the four major histologic subgroups of lung cancer in Denmark. J Clin Oncol. 2003; 21:3035-3040.

19. Merrill RM, Henson DE, Barnes M. Conditional survival among patients with carcinoma of the lung. Chest. 1999; 116:697-703.

20. Moller MB, Pedersen NT, Christensen BE. Conditional survival of patients with diffuse large B-cell lymphoma. Cancer. 2006; 106:2165-2170.

21. Mayo SC, Nathan H, Cameron JL, Olino K, Edil BH, Herman JM, Hirose K, Schulick RD, Choti MA, Wolfgang CL, Pawlik TM. Conditional survival in patients with pancreatic ductal adenocarcinoma resected with curative intent. Cancer. 2012; 118:2674-2681.
22. Henson DE, Ries LA, Carriaga MT. Conditional survival of 56,268 patients with breast cancer. Cancer. 1995; $76: 237-242$.

23. Efird JT, O'Neal WT, Camargo GA, Davies SW, O’Neal JB, Kypson AP. Conditional survival of heart failure patients after coronary artery bypass grafting. J Cardiovasc Med (Hagerstown). 2014; 15:498-503.

24. Zabor EC, Gonen M, Chapman PB, Panageas KS. Dynamic prognostication using conditional survival estimates. Cancer. 2013; 119:3589-3592.

25. Asrani SK, O'Leary JG. Acute-on-chronic liver failure. Clin Liver Dis. 2014; 18:561-574.

26. Romero-Gomez M, Montagnese S, Jalan R. Hepatic encephalopathy in patients with acute decompensation of cirrhosis and acute-on-chronic liver failure. J Hepatol. 2015; 62:437-447.

27. Malinchoc M, Kamath PS, Gordon FD, Peine CJ, Rank J, ter Borg PC. A model to predict poor survival in patients undergoing transjugular intrahepatic portosystemic shunts. Hepatology. 2000; 31:864-871.

28. Hieke S, Kleber M, Konig C, Engelhardt M, Schumacher M. Conditional Survival: A Useful Concept to Provide Information on How Prognosis Evolves over Time. Clin Cancer Res. 2015; 21:1530-1536.

29. Ferenci P, Lockwood A, Mullen K, Tarter R, Weissenborn K, Blei AT. Hepatic encephalopathy-definition, nomenclature, diagnosis, and quantification: final report of the working party at the 11th World Congresses of Gastroenterology, Vienna, 1998. Hepatology. 2002; 35:716-721.

30. Wai CT, Greenson JK, Fontana RJ, Kalbfleisch JD, Marrero JA, Conjeevaram HS, Lok AS. A simple noninvasive index can predict both significant fibrosis and cirrhosis in patients with chronic hepatitis C. Hepatology. 2003; 38:518-526.

31. EASL clinical practice guidelines on the management of ascites, spontaneous bacterial peritonitis, and hepatorenal syndrome in cirrhosis . J Hepatol. 2010; 53:397-417.

32. Arroyo V, Gines P, Gerbes AL, Dudley FJ, Gentilini P, Laffi G, Reynolds TB, Ring-Larsen H, Scholmerich J. Definition and diagnostic criteria of refractory ascites and hepatorenal syndrome in cirrhosis. International Ascites Club. Hepatology. 1996; 23:164-176.

33. Pugh RN, Murray-Lyon IM, Dawson JL, Pietroni MC, Williams R. Transection of the oesophagus for bleeding oesophageal varices. Br J Surg. 1973; 60:646-649. 